\title{
Galactic X-ray binary jets
}

\author{
Elena Gallo
}

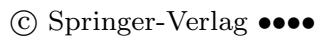

\begin{abstract}
With their relatively fast variability time-scales, Galactic X-ray binaries provide an excellent laboratory to explore the physics of accretion and related phenomena, most notably outflows, over different regimes. After comparing the phenomenology of jets in black hole systems to that of neutron stars, here I discuss the role of the jet at very low Eddington ratios, and present preliminary results obtained by fitting the broadband spectral energy distribution of a quiescent black hole binary with a 'maximally jet-dominated' model.
\end{abstract}

Keywords X-rays: binaries - radiation mechanisms: general - stars: individual (A0620-00)

\section{Black holes}

A key observational aspect of X-ray binary jets is their synchrotron radio emission. In black hole systems, radiatively inefficient, hard X-ray states (McClintock \& Remillard 2006; Homan \& Belloni 2005) are associated with flat/slightly inverted radio-to-mm spectra and persistent radio flux levels (Fender 2001). In analogy with compact extragalactic radio sources (Blandford \& Königl 1979), the flat spectra are thought to be due to the superposition of a number of peaked synchrotron spectra generated along a conical outflow, or jet, with the emitting plasma becoming progressively more transparent at lower frequencies as it travels away from the jet base. The jet interpretation has been confirmed by high resolution radio maps of two hard state black hole binaries (BHBs): Cygnus X-1 (Stirling et al. 2001) and GRS 1915+105 (Dhawan et al. 2000) are

Elena Gallo

Chandra Fellow

University of California, Santa Barbara, CA 93106, USA both resolved into elongated radio sources on milliarcsec scales - that is tens of A.U. - implying collimation angles smaller than a few degrees. Even though no collimated radio jet has been resolved in any BHB emitting X-rays below a few per cent of the Eddington limit, it is widely accepted, by analogy with the two above-mentioned systems, that the flat radio spectra associated with unresolved radio counterparts of X-ray binaries are originated in conical outflows.

Radiatively efficient, thermal dominant (high/soft) $\mathrm{X}$-ray states, on the contrary, are associated with no detectable core radio emission (Fender et al. 1999); as the radio fluxes drop by a factor up to 50 with respect to the hard state (e.g. Corbel \& Fender 2002, Corbel et al. 2004), this is generally interpreted as the physical suppression of the jet taking place over this regime.

Transient ejections of optically thin radio plasmons moving away from the binary core in opposite directions are often observed as a result of bright radio flares associated with hard-to-thermal X-ray state transitions. As proven by the case of e.g. GRS 1915+105 (Mirabel \& Rodríguez 1998), the same source can produce either kind of jets, steady/partially self-absorbed, and transient/optically thin, depending on the accretion regime.

\subsection{A unified model for black hole X-ray binaries}

Fender, Belloni \& Gallo (2004) have addressed the issue of whether the steady and transient jets of BHBs have a different origin or are somewhat different manifestations of the same phenomenon, showing that: i) the power content of the steady and transient jets are consistent with a monotonically increasing function of $L_{X}$; ii) the measured bulk Lorentz factors of the transient jets seem to be higher than those inferred for the steady jets. Based upon these arguments, the first unified model for the jet/accretion coupling in BHBs has been proposed. The key idea is that, as the thin disk inner boundary moves closer to the hole (hard-to-thermal 
state transition), the escape velocity from the inner regions increases. As a consequence, the steady jet bulk Lorentz factor rises sharply, causing the propagation of an internal shock through the slower-moving outflow in front of it. One typical cycle (lasting days to years, depending on the source) can be described as follows: the system is in the hard X-ray state, producing a steady jet. At some point $L_{\mathrm{X}} / L_{\mathrm{Edd}}$ increases above a few per cent while the X-ray spectrum softens, i.e. the accretion disk inner boundary is moving closer and closer to the $\mathrm{BH}$, resulting in an increase of the escape velocity. A sudden ejection of hot electrons (perhaps coupled with baryons) with high bulk Lorentz factor causes the propagation of a shock throughout the pre-existing outflow, and finally disrupts it. Eventually, the result of this shock is what we observe as a post-outburst, optically thin radio plasmon. No emission from the steady jet is detected until the inner disk recedes once more, in which case a new cycle begins.

However, there are at least a couple of recent results that might challenge some of the premises the proposed unified scheme is based on. The first one is the notion that, for the internal shock scenario to be at work and give rise to the bright radio flare at the state transition, whatever is ejected must have a higher velocity with respect to the pre-existing hard state steady jet. From an observational point of view, this was supported, on one side, by the lower limits on the transient jets' Lorentz factors, typically higher than 2 (Fender 2003), and, on the other hand, by the relative small scatter about the radio/X-ray correlation in hard state BHBs (Gallo, Fender \& Pooley 2003). The latter has been challenged on theoretical grounds (Heinz \& Merloni 2004), while a recent work (Miller-Jones et al. 2006) has demonstrated that, from an observational point of view, the average Lorentz factors do not differ substantially between hard and transient jets (albeit the estimated Lorentz factors rely on the assumption of no lateral confinement).

In addition, recent high statistics X-ray observations of hard state BHBs undergoing outburst (Miller et al. 2006; Rykoff et al. 2007) suggest that a cool, thin accretion disk extends already near to the innermost stable circular orbit (ISCO) already during the bright phases of the hard state, that is prior to the top horizontal brunch in the top panel of Figure 7 in Fender, et al. (2004). This would challenge the hypothesis of a sudden deepening of the inner disk potential well as the cause of a high Lorentz factor ejection. Possibly, whether the inner disk radius moves close to the hole prior or during the softening of the X-ray spectrum does not play such a crucial role in terms of jet properties; if so, then the attention should be diverted to a different component, such as the presence/absence, or the size
(Homan et al. 2001) of a Comptonizing corona (which could in fact coincide with the very jet base; Markoff, Nowak \& Wilms 2005).

Finally, much work needs to be done in order to test the consistency of the internal shock scenario as a viable mechanism to account for the observed changes in the radio properties, given the various observational and theoretical constraints (such as emissivities, radio/infrared delays, cooling times, mass outflow rates, etc.).

One of the most interesting aspects of this proposed scheme - assuming that is correct in its general principles - is obviously its possible application to supermassive BHs in Active Galactic Nuclei (AGN), and the possibility to mirror different X-ray binary states into different classes of AGN: radio loud vs. radio quiet, LLAGN, FRI, FRII etc.. The interested reader is referred to Körding, Jester \& Fender (2006).

\section{Neutron stars}

Low magnetic field, 'atoll-type' neutron star X-ray binaries (see van der Klis 2006 for a classification) share many X-ray spectral and timing properties with BHBs and show two distinct X-ray states, which can be directly compared to the hard and thermal state of BHBs: the reader is referred to Migliari \& Fender (2006) for a comprehensive work of correlated radio and X-ray properties of neutron stars (NSs). The main differences/similarities can be summarized as follows: as for the BHBs, below a few per cent of their Eddington luminosity, NSs power steady, self-absorbed jets. Transient optically thin plasmons are ejected at higher X-ray luminosities, in response to rapid X-ray state changes. However, NSs are less 'radio loud' than BHBs. At a given $L_{\mathrm{X}} / L_{\mathrm{Edd}}$, the difference in radio luminosity is typically a factor $\sim 30$, which can not be accounted for by the mass ratio alone. In contrast to $\mathrm{BHs}$, atolls have been detected in the radio band while in the soft $\mathrm{X}$-ray state, suggesting that the jet quenching in diskdominated states may not be so extreme. Like in the BHBs, the radio luminosity of hard state NSs scales non-linearly with the X-ray luminosity (see Section 3), even though the slope of radio-to-X-ray correlation albeit the low statistics- appears to be steeper for the NSs $\left(L_{\text {radio }} \propto L_{\mathrm{X}}^{b}\right.$, with $b \simeq 0.7$ for the BHs, and $b \simeq 1.4$ for the NSs; see Körding, Fender \& Migliari 2006 for an interpretation).

High magnetic field, 'Z-type' NSs, being persistently close to the Eddington accretion rate, behave similar to the rapidly varying BHB GRS $1915+105$. The 
role played by the NS dipole magnetic field in producing/inhibiting the jet production mechanism is far from being clear, although it is generally assumed that the higher the magnetic field, the lower the jet power. Further radio observations of X-ray pulsars and ms accreting X-ray pulsars are definitely needed in order to place stronger observational constraints.

Overall, these results indicate that, at the zeroth order, the process of continuous jet formation works similarly in accreting BHs and (at least certain) NSs. Even though quantitative differences remain to be explained, such as the relative dimness of neutron star jets with respect to $\mathrm{BH}$ jets, this indicates that the presence of an accretion disk coupled to an intense gravitational field would be the necessary and sufficient ingredients for steady relativistic jets to be formed, regardless of the presence/absence of an event horizon. In addition, it is worth reminding that the most relativistic Galactic jet source observed so far is a NS (Fender et al. 2004), contrary to what it might be expected according to the so called 'escape-velocity paradigm'.

\section{Luminosity Correlations}

\section{Radio/X-ray}

In a first attempt to quantify the relative importance of jet vs. disk emission in BHBs, Gallo et al. (2003) collected quasi-simultaneous radio and $\mathrm{X}$-ray observations of ten hard state sources. This study established the presence of a tight correlation between the X-ray and the radio luminosity, of the form $L_{\mathrm{R}} \propto L_{\mathrm{X}}^{0.7 \pm 0.1}$, first quantified for GX 339-4 (Corbel et al. 2003). The correlation extends over more than 3 orders of magnitude in $L_{\mathrm{X}}$ and breaks down around 2 per cent of the Eddington X-ray luminosity, above which the sources enter the thermal dominant state, and the core radio emission drops below detectable levels. Given its non-linearity, the ratio radio-to-X-ray luminosity increases towards quiescence (i.e. below a few $10^{-6} L_{\mathrm{Edd}}$ ). Thanks to the quite large degree of uncertainty about the overall structure of the accretion flow in this regime, this has led to the hypothesis that the total power output of quiescent BHBs could be dominated by a radiatively inefficient outflow, rather than by the local dissipation of gravitational energy in the accretion flow (Fender, Gallo \& Jonker 2003; Körding, Fender \& Migliari 2006). Simultaneous radio/X-ray observation of the nearest quiescent BHB A0620-00 have confirmed that the nonlinear correlation holds down to Eddington ratios as low as $10^{-8}$ (Gallo et al. 2006). Nevertheless, many

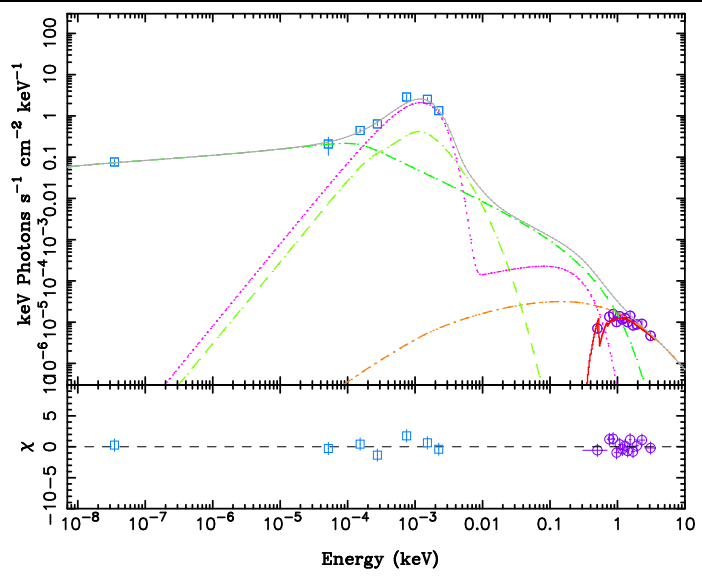

Fig. 1.- Fit to broadband SED of the BHB A062000 with the 'maximally jet-dominated model'. The symbols represent the data, while the solid red line is the model fit in detector space. Solid (gray): total spectrum; Dot-long-dashed (light green): preacceleration inner jet synchrotron emission; Dot-longdashed (darker green): post-acceleration outer jet synchrotron; Dot-dash-dash (orange): Compton emission from the inner jet; Dot-short-dash (magenta): thermal multicolor-blackbody disk model plus single blackbody representing the star. From Gallo et al. (submitted to ApJ).

outliers have been recently been found at higher luminosities (see Gallo 2007, Xue \& Cui 2007, and references therein), casting doubts on the universality of this scaling, and the possibility of relying on the best-fitting relation for estimating other quantities, such as distance or BH mass.

\section{Optical-Infrared/X-ray}

The infrared (IR) spectra of BHBs with a low mass donor star are likely shaped by a number of competing emission mechanisms, most notably: reprocessing of accretion-powered X-ray and ultraviolet photons, either by the donor star surface or by the outer accretion disk, direct thermal emission from the outer disk, and non-thermal synchrotron emission from a relativistic outflow. Russell et al. (2006) have collected all the available quasi-simultaneous optical and near-IR data of a large sample of Galactic X-ray binaries over different X-ray states. The optical/near-IR (OIR) luminosity of hard/quiescent BHBs is found to correlate with the X-ray luminosity to the power $\sim 0.6$, consistent with the known radio/X-ray correlation slope down to $10^{-8} L_{\text {Edd }}$ (Gallo et al. 2006). Combined with the fact that the near-IR emission is largely suppressed in the thermal-dominant state, this leads to the conclusion that, for the BHBs, the break to the optically thin 
portion would take place in the mid-IR $(2-40 \mu \mathrm{m})$. A similar correlation is found in low-mass neutron stars (NSs) in the hard state. By comparing the observed relations with those expected from models of a number of emission processes, Russell et al. (2006) are able to constrain the mean OIR contributions to the spectral energy distribution (SED) for the different classes of X-ray binaries. They conclude that the jets are contributing 90 per cent of the near-IR emission at high luminosities in the hard state of BHBs. The optical emission could have a substantial (up to 75 per cent) jet contribution; however, the optical SEDs show a thermal spectrum indicating X-ray reprocessing in the disk dominates in this regime. In contrast, X-ray reprocessing dominates the OIR in hard state NSs, with possible contributions from the jets and the viscously heated disk only at high luminosities.

\section{The role of the jet in quiescence}

There is evidence from large scale-structures that the jets' mechanical power is comparable to the bolometric X-ray luminosity in some hard state BHB sources (e.g. Russell et al. 2007). However, even for the highest quality SED, disentangling the relative contributions of inflow vs. outflow to the radiation spectrum and global accretion energy budget can be quite challenging, as illustrated by the emblematic case of XTE $\mathrm{J} 1118+480$ in McClintock et al. (2003) and Markoff et al. (2003). Estimates of the total jet power based on its radiation spectrum depend crucially on the assumed frequency at which the flat, partially self-absorbed spectrum turns and becomes optically thin, as the jet 'radiative efficiency' depends ultimately on the location of the high-energy cutoff induced by the higher synchrotron cooling rate of the most energetic particles. Once again, this quantity has proved hard to measure. Indeed, from a theoretical point of view, the 'break frequency', here defined as the frequency at which the partially self-absorbed jet becomes optically thin, is inversely proportional to the $\mathrm{BH}$ mass: as jet spectral breaks are often observed in the $\mathrm{GHz} /$ sub-mm regime in active nuclei, they are expected to occur in the IRoptical band for $10^{5-7}$ times lighter objects. We know however from observations of GX 339-4, the only BHB where the optically thin jet spectrum has been perhaps observed (Corbel \& Fender 2002, Homan et al. 2005), that the exact break frequency can vary with the overall luminosity, possibly reflecting changes in the magnetic field energy density, particle density and mass loading at the jet base. Determining the location of the jet break as a function of the bolometric luminosity is important to assess the synchrotron contribution to the hard X-ray band. As an example, that the optically thin jet IR-emission in GX 339-4 connects smoothly with the hard X-ray power law has led to challenge the 'standard' Comptonization scenario for the hard X-ray state (Markoff et al. 2001), whereas recent Spitzer observations of the ultra-compact neutron star X-ray binary 4U 0614+091 (Migliari et al. 2006) revealed that the break frequency must take place in the far-IR in this system, effectively ruling out a synchrotron origin for the X-ray power law.

\subsection{Case study: A0620-00}

The role of the jet -if any- is especially interesting at very low luminosities, in the so called 'quiescent' regime, i.e. below a few $10^{-6} L_{\mathrm{Edd}}$. Steady jets appear to survive down to quiescent X-ray luminosities (Gallo et al. 2006), even though sensitivity limitations on current radio telescopes make it extremely difficult to reach the signal-to-noise ratios required to assess their presence for low luminosity systems further than $2 \mathrm{kpc}$ or so. In spite of the large degree of uncertainty on the overall geometry of the accretion flow in this regime, there is general agreement that the Xray emission in quiescent $\mathrm{BHBs}$ comes from high-energy electrons near the $\mathrm{BH}$, the disagreement comes about in: i) attributing the emission to outflowing vs. inflowing electrons; ii) modeling the electron distribution as thermal vs. non-thermal (or hybrid; McClintock et al. 2003). The SEDs of quiescent BHBs, as well as low-luminosity AGN are often examined in the context of the advection-dominated accretion flow solution (Narayan \& Yi 1994), whereby the low X-ray luminosities would be due to a highly reduced radiative efficiency, and most of the liberated accretion power disappears into the horizon. Alternatively, building on the work by Falcke \& Biermann (1995) on AGN jets, a jet model has been proposed for hard state BHBs (see Markoff et al. 2005 for the latest version). The model is based upon four assumptions: 1) the total power in the jets scales with the total accretion power at the innermost part of the accretion disk, $\left.\dot{m} c^{2}, 2\right)$ the jets are freely expanding and only weakly accelerated via their own internal pressure gradients only, 3) the jets contain cold protons which carry most of the kinetic energy while leptons dominate the radiation and 4) some fraction of the initially quasi-thermal particles are accelerated into power-law tails.

Figure 1 shows a fit to the SED of A0620-00, the lowest Eddington-ratio BHB with a known radio counterpart $\left(L_{\mathrm{X}} / L_{\mathrm{Edd}} \simeq 10^{-8}\right)$ with such a 'maximally jet-dominated' model (Gallo et al. 2007, submitted to ApJ). This is the first time that such a complex model 
is applied in the context of quiescent BHBs, and with the strong constraints on the jet break frequency cutoff provided by the Spitzer data in the mid-IR regime. The model is most sensitive to the fitted parameter $N_{\mathrm{j}}$, which acts as a normalization, though it is not strictly equivalent to the total power in the jets (see discussion in MNW05). It dictates the power initially divided between the particles and magnetic field at the base of the jet, and is expressed in terms of a fraction of $L_{\mathrm{Edd}}$. Once $N_{\mathrm{j}}$ is specified and conservation is assumed, the macroscopic physical parameters along the jet are determined assuming that the jet power is roughly shared between the internal and external pressures. The radiating particles enter the base of the jet where the bulk velocities are lowest, with a quasithermal distribution. Starting at location $z_{\text {acc }}$ in the jets, a free parameter, a fraction $85 \%$ of the particles are accelerated into a powerlaw with index $p$, also a fitted parameter. The maximum energy of the accelerated leptons is calculated by setting the acceleration rate to the local cooling rates from synchrotron and inverse Compton radiation at $z_{\text {acc }}$. If the acceleration process is diffusive Fermi acceleration, the acceleration rate depends on the factor $f=\frac{\left(u_{\mathrm{acc}} / c\right)^{2}}{f_{s c}}$, where $u_{\mathrm{acc}}$ is the shock speed relative to the bulk plasma flow, and $f_{\mathrm{sc}}$ is the ratio of the scattering mean free path to the gyro-radius. Because neither plasma parameter is known, we fit for their combined contribution $f$, which thus reflects the efficiency of acceleration. We find $f$ to be around two orders of magnitude lower for A0620-00 than in higher luminosity sources. This 'weak acceleration' scenario is reminiscent of the Galactic Center super-massive BH Sgr A*. Within this framework, the SED of Sgr $A^{*}$ does not require a power law of optically thin synchrotron emission after the break from its flat/inverted radio spectrum (Falcke \& Markoff 2000). Therefore, if the radiating particles have a power-law distribution, it must be so steep as to be indistinguishable from a Maxwellian in the optically thin regime. In this respect, they must be only weakly accelerated. Here we have shown that something similar, albeit less extreme, is occurring in the quiescent BHB A0620-00; either scenario implies that acceleration in the jets is inefficient at $10^{-9}-10^{-8} L_{\mathrm{Edd}}$.

This work is supported by NASA through Chandra Postdoctoral Fellowship grant number PF5-60037, issued by the Chandra X-Ray Center, which is operated by the Smithsonian Astrophysical Observatory for NASA under contract NAS8-03060. I wish to thank the organizers for the interesting conference, as well as for the memorable excursion to Tidbinbilla.

\section{References}

Blandford R. D., Königl A., 1979, ApJ, 232, 34

Corbel S., Fender R. P., Tomsick J. A., Tzioumis A., Tingay S., 2004, ApJ, 617, 1272

Corbel S., Nowak M., Fender R. P., Tzioumis A. K., Markoff S., 2003, A\&A, 400, 1007

Corbel S. \& Fender R., 2002, ApJ, 573, L35

Dhawan V., Mirabel I. F., Rodríguez L. F., 2000, ApJ, 543, 373

Falcke H. \& Markoff S., 2000, A\&A, 362, 113

Falcke H. \& Biermann P. L., 1996, A\&A, 308, 321

Fender R. P., 2006, in Lewin W. H. G., van der Klis M., eds, Compact Stellar X-Ray Sources. Cambridge Univ. Press, Cambridge

Fender R. et al. , 2004, Nature, 427, 222

Fender R. P., Belloni T., Gallo E., 2004, MNRAS, 355, 1105

Fender R., 2003, MNRAS, 340, 1353

Fender R. P., Gallo E., Jonker P. G., 2003, MNRAS, 343, L99

Fender R. P., 2001, MNRAS, 322, 31

Fender R. P. et al. , 1999, ApJ, 519, L165

Gallo E., 2007, in Proc. of 'The Multicoloured Landscape of Compact Objects and their Explosive Origins', AIP Conf. Proc. (astro-ph/0702126)

Gallo E. et al. , 2006, MINRAS, 370, 1351

Gallo E., Fender R. P., Pooley G. G., 2003, MNRAS, 344, 60

Heinz S. \& Merloni A., 2004, MNRAS, 355, L1

Homan J. et al. , 2001, ApJS, 132, 377

Homan J., Buxton, M., Markoff, S., Bailyn, C., Nespoli, E., Belloni, T., 2005, ApJ, 624, 295

Homan J. \& Belloni T., 2005, A\&SS, 107

Hynes R. et al. , 2006, ApJ, 651, 401

Klis van der, M., 2006, in Lewin W. H. G., van der Klis M., eds, Compact Stellar X-Ray Sources. Cambridge Univ. Press, Cambridge

Körding E., Jester S., Fender R., 2006, MNRAS, 372, 1366

Körding E., Fender R., Migliari S., 2006, MNRAS, 369, 1451

Markoff S., Nowak M. A., Wilms J., 2005, ApJ, 635, 1203

Markoff S., Nowak, M., Corbel, S., Fender, R., Falcke, H., 2003, A\&A, 397, 645

Markoff S., Falcke H., Fender R., 2001, A\&A, 372, L25

Miller J., Homan, J., Steeghs, D., Rupen, M., Hunstead, R. W., Wijnands, R., Charles, P. A., Fabian, A. C., 2006a, ApJ, 653, 525

Miller-Jones J., Fender R., Nakar E., 2006, MNRAS, 367, 1432

McClintock J. E., Remillard R. A., 2006, in Lewin W. H. G., van der Klis M., eds, Compact Stellar X-Ray Sources. Cambridge Univ. Press, Cambridge

McClintock J. E. et al. , 2003, ApJ, 593, 435

Migliari S., Tomsick, J., Maccarone, T., Gallo, E., Fender, R., Nelemans, G., Russell, D., 2006, ApJ, 643, L41

Migliari S. \& Fender R., 2006, MNRAS, 366, 79

Mirabel I. F., Rodríguez L. F., 1998, Nature, 392, 673

Narayan R., Yi I. 1994, ApJ, 428, L13

Russell D., Fender R., Gallo E., Kaiser C., 2007, MNRAS, in press (astro-ph/0701645)

Russell, D., Fender, R., Hynes, R., Brocksopp, C., Homan, J., Jonker, P., Buxton, M., 2006, MNRAS, 371, 1334

Rykoff E., Miller J., Steeghs D., Torres M., 2007, submitted to ApJ (astro-ph/0703497)

Stirling A. M. et al. , 2001, MINRAS, 327, 1273

Xue Y. \& Cui W., 2007, A\&A, in press (astro-ph/0702684)

This 2-column preprint was prepared with the AAS LATEX macros $\mathrm{v} 5.2$. 